\begin{tabular}{|c|c|c|}
\hline Beitr. Ent. & Keltern & ISSN 0005-805X \\
\hline $\mathbf{6 2}(2012) 2$ & S. $361-374$ & 20.12 .2012 \\
\hline
\end{tabular}

\title{
Zur Kenntnis der Scirtidae des Iran mit Beschreibung je einer neuen Art aus den Gattungen Cyphon PaYKull, 1799 und Elodes LATREILLE, 1796
}

\section{(Coleoptera)}

\section{(175. Beitrag zur Kenntnis der Scirtidae)}

Mit 20 Figuren und 4 Tabellen

\section{Bernhard Klausnitzer}

\section{Zusammenfassung}

Aus dem Iran werden zwei neue Arten beschrieben: Cyphon pareuoplus n. sp. und Elodes unicolor n. sp. Bisher sind aus diesem Land 16 Arten der Familie Scirtidae bekannt, von denen sieben bisher nur aus dem Iran nachgewiesen wurden, drei haben ein großes Areal, die übrigen sind kaspische, möglicherweise zum Teil auch syrische Faunenelemente.

\section{Summary}

Two new species are described from Iran: Cyphon pareuoplus n. sp. and Elodes unicolor n. sp. So far 16 species are known from this country, of which seven have until now only been found in Iran, three have a large range, and the others are Caspian or possibly in part Syrian faunal elements.

\section{Einleitung}

Die Kenntnis der Scirtidenfauna des Iran ist nach wie vor sehr lückenhaft. Die Größe des Landes, die Vielfalt der Lebensräume und der Einfluss verschiedener Zentren der Paläarktis, wohl auch der Orientalischen und der Afrotropischen Region, lassen eine deutlich höhere Artenzahl als bisher bekannt erwarten. Bisher wurden im Iran 14 Arten aus 5 Gattungen nachgewiesen, zu denen jetzt zwei neue Arten hinzu kommen. Zusätzlich werden Funddaten der von Andreas Pütz im Iran gesammelten Tiere bekannt gegeben.

Einige auf den Etiketten angegebene Ortsnamen aus früheren Untersuchungen (KLAusNitzer 1975, 1981, Nyноцм 1976) konnten nicht sicher lokalisiert werden.

\section{Anmerkungen zu einer zoogeografischen Analyse}

In der folgenden Tabelle 1 werden die bisherigen Kenntnisse über das Vorkommen der Scirtidae im Iran und den angrenzenden Ländern zusammengefasst. Die hohe Artenzahl in der östlichen Türkei einerseits und die sehr niedrigen Artenzahlen vor allem in Afghanistan und Pakistan andererseits gehen auf den sehr unterschiedlichen Durchforschungsgrad (geringe Sammeltätigkeit) 
Tab. 1: Vergleich des Arteninventars des Iran mit den angrenzenden Ländern (zusammenfassende Angaben nach Klausnitzer 2006, 2009a, b, 2010, 2012, im Druck). Abkürzungen: öTR = östliche Türkei, AR = Armenien, $\mathrm{AB}=$ Aserbaidschan, $\mathrm{IQ}=$ Irak, $\mathrm{IN}=$ Iran, $\mathrm{TM}=$ Turkmenistan, $\mathrm{AF}=$ Afghanistan, $\mathrm{PA}=$ Pakistan.

\begin{tabular}{|c|c|c|c|c|c|c|c|c|}
\hline Art & öTR & AR & $\mathrm{AB}$ & IQ & IN & TM & $\mathrm{AF}$ & PA \\
\hline Cyphon abeillei BOURGEOIs, 1884 & + & & & & & & & \\
\hline Cyphon algenib KLAUSNITZER 2003 & & & & & + & & & \\
\hline Cyphon arietizans NyHOLM, 1970 & + & + & & & & & & \\
\hline Cyphon buceros Nyноцм, 1949 & + & + & + & & & & & \\
\hline Cyphon caspicus KlausNitzer, 1975 & & & + & & & & & \\
\hline Cyphon coarctatus PAYKULL, 1799 & & & & & & + & & \\
\hline Cyphon dinolabis Nуноцм, 1970 & + & & & & & & & \\
\hline Cyphon euoplus Nyноцм, 1970 & + & & & & & & & \\
\hline Cyphon euryceros Nyноцм, 1970 & + & & & & & & & \\
\hline Cyphon laevipennis TOURNIER, 1868 & + & + & + & + & + & + & + & \\
\hline Cyphon lepidulus Nyноцм, 1968 & + & & + & + & + & & & \\
\hline Cyphon longispinus NYноцм, 1968 & + & & & & & & & \\
\hline Cyphon longiusculus Nyноцм, 1970 & + & & + & & + & & & \\
\hline Cyphon ochraceus STEPHENS, 1830 & + & & & & & & & \\
\hline Cyphon osellai KLAUSNITZER, 1989 & + & & & & & & & \\
\hline Cyphon padi (Linnaeus, 1758) & + & & & & & & & \\
\hline Cyphon palustris C. G. Thомson, 1855 & + & + & & & + & & & \\
\hline Cyphon pareuoplus n. sp. & & & & & + & & & \\
\hline Cyphon perses Nyноцм, 1976 & & & & & + & & & \\
\hline Cyphon ponticus Nуноцм, 1976 & + & & & & & & & \\
\hline Cyphon pubescens (FABRICIUS, 1792) & + & & & & & & & \\
\hline Cyphon quadrum KLAUSNITZER, 1980 & & + & + & & & & & \\
\hline Cyphon reitteri KLAUSNITZER, 1976 & & & + & & & & & \\
\hline Cyphon ruficeps TOURNIER, 1868 & + & & & & & & & \\
\hline Cyphon scurrula NyноLм, 1970 & + & & & & & & & \\
\hline Cyphon simplex KLAUSNITZER, 1981 & & & & & + & & & \\
\hline Cyphon wittmeri NYHOLM, 1970 & + & & & & & & & \\
\hline Elodes akbesiana PIC, 1898 & + & & & & & & & \\
\hline Elodes eberti KLAUSNITZER, 1970 & + & & + & & + & & & \\
\hline Elodes flavoangularis KLAUSNITZER, 1990 & + & & & & & & & \\
\hline Elodes improvisa KLAUSNITZER, 1990 & + & & & & & & & \\
\hline Elodes luteata KLAUSNITZER, 1979 & + & & & & & & & \\
\hline Elodes megalopa KLAUSNITZER, 2002 & + & & & & & & & \\
\hline Elodes microps KLAUSNITZER, 2002 & + & & & & & & & \\
\hline Elodes minuta (LinnaEus, 1767) & + & & & & & & & \\
\hline Elodes orientalis IABLOKOFF-KHNZORIAN, 1973 & & & & & + & & & \\
\hline Elodes persica KLAUSNITZER, 1975 & & & & & + & & & \\
\hline Elodes persimilis KLAUSNITZER, 2002 & + & & & & & & & \\
\hline Elodes pseudominuta KLAUSNITZER, 1971 & + & & & & & & & \\
\hline Elodes pseudoscutellaris KLAUSNITZER, 1973 & & + & & & & & & \\
\hline
\end{tabular}




\begin{tabular}{|l|c|c|c|c|c|c|c|c|}
\hline \multicolumn{1}{|c|}{ Art } & öTR & AR & AB & IQ & IN & TM & AF & PA \\
\hline Elodes sieberi KLAUSNITZER, 1973 & + & & & & & & & \\
\hline Elodes tegminis KLAUSNITZER, 2012 & & & & & & & & + \\
\hline Elodes tournieri KIESENWETTER, 1871 & + & & & & & & & \\
\hline Elodes unicolor n. sp. & & & & & + & & & \\
\hline Elodes venustula KLAUSNITZER, 2002 & + & & & & & & & \\
\hline Hydrocyphon consolatorius KLAUSNITZER, 1990 & & & & & + & & & \\
\hline Hydrocyphon deflexicollis (P. W. J. MÜLLER, 1821) & + & & & & & & & \\
\hline Hydrocyphon finitimus NYHOLM, 1977 & + & + & & & & & & \\
\hline Hydrocyphon interrogationis KLAUSNITZER, 1980 & & & & & & & & + \\
\hline Hydrocyphon kopetdaghensis RUTA, 2007 & & & & & & + & & \\
\hline Hydrocyphon nuristanicus KLAUSNITZER, 2004 & & & & & & & + & \\
\hline Hydrocyphon rivulorum NYHOLM, 1977 & + & & & & & & & \\
\hline Hydrocyphon segrex NYHOLM, 1972 & + & & + & & + & & & \\
\hline Hydrocyphon vicinans NYHOLM, 1972 & + & & & & & & & \\
\hline Hydrocyphon yoshitomii KLAUSNITZER, 2002 & & & & & & & & + \\
\hline Hydrocyphon zwicki KLAUSNITZER, 2011 & & & & & & & & + \\
\hline Microcara luteicornis REITTER, 1888 & + & & + & & + & & & \\
\hline Odeles armilabris (NYHOLM, 1974) & + & & & & & & & \\
\hline Prionocyphon ornatus ABEILLE DE PERRIN, 1881 & + & & + & & & & & \\
\hline Scirtes haemisphaericus (LINNAEUS, 1767) & + & & & & & & & \\
\hline Scirtes orbicularis (PANZER, 1793) & + & & + & & + & & & \\
\hline Summe & 42 & 7 & 12 & 2 & 16 & 3 & 2 & 4 \\
\hline
\end{tabular}

zurück. Die Artenzahlen der einzelnen Länder können deshalb nicht miteinander verglichen werden. Dennoch wird ein gewisser Eindruck von der voraussichtlich vorhandenen Vielfalt der Scirtidenfauna des Iran vermittelt.

\subsection{Exklusive Arten}

Fünf Arten der Scirtidae wurden bisher ausschließlich aus dem Iran nachgewiesen. Hinzu kommen die beiden hier neu beschriebenen Arten. Cyphon algenib und C. perses haben offenbar eine hyrkanische Verbreitung, sind also Endemiten am Südrand des Kaspischen Meeres. De LatTin (1967) hat den hyrkanischen Raum noch nicht differenziert, er ist Teil des kaspischen Verbreitungstyps sensu De LatTin (Frisch in litt.).

\section{Cyphon algenib KLAUSNITZER, 2003}

Die Art wurde unter dem Namen Cyphon simulans Klausnitzer, 1990 beschrieben. Der locus typicus liegt im Norden Irans: „Sheyk Mahalley“. Sheykh Mahalley [36 27' N; 52 34' E] liegt in der Provinz Māzandarān südlich des Kaspischen Meeres.

Neues Material: $10^{\star}$, IRAN, Prov. Mazandaran [IR08-22], Tonekabon County, Elburz Mts., N-Slope, 4,5 km SW Khanian, Sehezar forest, leaves debris, sifted, small stream, $942 \mathrm{~m}$, 05.VI.2008, 36³2'61,7" N; 5049'89,2" E, leg. A. Pütz, det. B. Klausnitzer. 


\section{Cyphon perses NyHOLM, 1976}

Die Art wurde aus „Assalam“ beschrieben (Nyноцм 1976). Dieser Fundort kann nicht sicher lokalisiert werden. Vermutlich handelt es sich um „Asalem“ in der Provinz Gilan (FrIsCH in litt.) Weitere Exemplare sind von folgenden Lokalitäten bekannt:

KLausnitzer (1990): „Gīlān, Nav’s Valley“. - Die Provinz Gīlān liegt am südwestlichen Ufer des Kaspischen Meeres. „Nav's Valley“ ist ein Flusstal im Talisch, in dem die Straße von Asalem nach Khalkhal verläuft und die Ortschaft Nav liegt.

Klausnitzer (1990): „Mazandaran, Delasm“. - Die Provinz Māzandarān liegt südlich des Kaspischen Meeres. Mit „Delasm“ ist „Dalasm“ = „Dalasam“ südlich Now Shahr (36 26 $40^{\prime}$ N; 51 $32^{\prime} 10^{\prime}$ E) gemeint. KLAusnitzer (2012): Māzandarān, 20 km SW Pol-e Sefid: 2 km NE Allahband, [3603'04" N; 5257'03" E]. - Der Fundort liegt im Elbursgebirge.

Neues Material: $10^{\star}$, IRAN, Prov. Mazandaran [IR08-01A], Sari County, Mohammadabad, Elburz Mts., N-Slope, 2,2 km NE Bendela, Fagus forest, 1533 m, 30.V.2008, 3604'06,6" N; 5309'57,8" E, leg. A. Pütz, det. B. Klausnitzer. 1 ơ $^{\star}$, IRAN, Prov. Mazandaran [IR08-04], Sari County, Mohammadabad, Elburz Mts., N-Slope, $1 \mathrm{~km}$ W Afra Chal, small stream, leaves, 520 m, 29.V.2008, 36²14'11,9" N; 53¹3'61,0" E, leg. A. Pütz, det. B. Klausnitzer.

\section{Cyphon simplex KLAUSNITZER, 1981}

Diese Art wurde aus „Emāmek“ beschrieben (locus typicus). Das Fundortetikett vermerkt noch die Lage des Ortes im Elbursgebirge, eine genaue Lokalisierung war nicht möglich. Das Areal von Cyphon simplex ist offenbar recht ausgedehnt.

KLausnitzer (1990): „Kordestān, Santeh“. - Die Provinz Kordestān liegt im Westen des Iran. „Santeh“ liegt östlich Saqqez und östlich Saheb bei 36 $10^{\prime} 0 "$ N; 46 $33^{\prime} 0^{\prime \prime}$ E.

Klausnitzer (1990): „Kordestān, S. de Dīvān Darreh“. - Dīvān Darreh hat die Koordinaten 3555' N; $47^{\circ} 01^{\prime} \mathrm{E}$.

Klausnitzer (1990): „Kordestān, S.-E. de Kal'eh Dju“. - „Kal’eh Dju“ liegt südlich Marivan bei 35²1' N; $46^{\circ} 17^{\prime} \mathrm{E}$.

KLAusnitzer (1990): „Kermānshāhāh, N. de Kermānshāh“. - Die Provinz Kermānshāhāh schließt südlich an Kordestān an. Kermānshāh hat die Koordinaten $34^{\circ} 18^{\prime}$ N; $47^{\circ} 03^{\prime}$ E.

Klausnitzer (1990): „Hamadān, Asadābād“. - Die Provinz Hamadān schließt östlich an Kordestān an. Asadābād hat die Koordinaten $34^{\circ} 47^{\prime} \mathrm{N}$; $48^{\circ} 06^{\prime}$ E.

KLausnitzer (1990): „Māzandarān, Nowshahr“. - Now Shahr liegt am Südrand des Kaspischen Meeres und hat die Koordinaten $36^{\circ} 38^{\prime}$ N; $51^{\circ} 30^{\prime}$ E.

\section{Elodes persica KLAUSNITZER, 1975}

Die Art wurde aus „Gole Lovae“ beschrieben. Gole Lovae liegt $80 \mathrm{~km}$ SSW von Gorgan in der Provinz Golestan.

KLAusnitzer (2012): Semnān, Straße zwischen Shāhrūd und Mojen: 2 km SE Tash, 2190 m [36³2'44" N; 54²0'06" E]. - Die Straße Shahrud - Mojen verläuft am Südabfall des Elburs.

Gole Lovae und Mojen liegen nahe beieinander am Nord- bzw. Südabfall des gleichen Gebirgsstockes, des Shah Kuh.

\section{Hydrocyphon consolatorius KLAUSNITZER, 1990}

Die Art wurde aus „Bīshāpor, Tangé Chogan“ beschrieben. Bīshāpor liegt in der Provinz Fārs im Zagrosgebirge und hat die Koordinaten $29^{\circ} 47^{\prime} \mathrm{N} ; 51^{\circ} 36^{\prime} \mathrm{E}$. 


\subsection{Kaspische Arten}

In der Definition des kaspischen Zentrums wird De LatTin (1967) gefolgt.

\section{Microcara luteicornis REITTER, 1888}

Das große Areal dieser Art umfasst außer den in Tabelle 1 angegebenen Ländern auch Georgien, Südrussland und die westliche Türkei.

KLAusnitzer (2012): Māzandarān, Nur [36³4'36" N; 5204'34" E].

Neues Material: 5 우 우, IRAN, Prov. Mazandaran [IR08-17], Chalus County, Elburz Mts., N-Slope, $7 \mathrm{~km} \mathrm{~N} \mathrm{Makarud,} \mathrm{small} \mathrm{stream,} \mathrm{Fagus} \mathrm{forest,} 1245$ m, 03.VI.2008, 36 35'64,9" N; 5109'55,4" E, leg. A. Pütz, det. B. Klausnitzer.

\subsection{Arten mit einem nach Osten ausgedehnten Areal}

\section{Elodes orientalis IABLOKOFF-KHNZORIAN, 1973}

Diese Art kommt auch in Tadschikistan vor (KLAusnitzer 2009b).

KLAusnitzer (2012): Hamadān, Moradbeyk valley (Kūh-e Alvand) [34²3'09" N; 48²9'01" E].

\subsection{Arten mit einem unklaren Verbreitungsbild}

\section{Elodes eberti KLAUSNITZER, 1970}

Das Areal von Elodes eberti ist nicht recht einzuschätzen. Der Nachweis in der Ukraine (Karpaten) ist ein Einzelfund (Klausnitzer 2008) und liegt weit entfernt von den übrigen Fundorten. Die Art wurde aus der Provinz Tehrān beschrieben, wo auch die übrigen Fundorte aus dem Iran liegen (KLausnitzer 1970, 1972, 1990).

Bei den im Folgenden genannten Arten könnte es sich um kaspische oder syrische Arten handeln. Die Funde im ostmediterranen Raum legen aber auch die Vermutung nahe, dass es sich um expansive pontomediterrane Elemente handeln könnte. Insgesamt liegen für eine zoogeografische Zuordnung zu wenige Daten vor.

\section{Cyphon lepidulus NyHOLM, 1968}

Diese Art kommt auch in Griechenland, auf Kreta und Zypern, in Syrien sowie der westlichen Türkei vor (KLAUSNitzer 2009a, 2012).

Klausnitzer (1990): „Kordestān, Marivān“. - Marivān hat die Koordinaten 35³1' N; 46¹0' E.

KLAusnitzer (1990): „Gīlān, Galūgāh, Bandar Pahlevi“. - Galūgāh liegt westlich Bandar-e-Anzali bei $37^{\circ} 29^{\prime} 58^{\prime \prime} \mathrm{N}$; 49 19'35" E. „Bandar Pahlevi“ wurde in „Bandar-e-Anzali“ unbenannt (Hafenstadt am Kaspischen Meer).

\section{Cyphon longiusculus NYHOLM, 1970}

Cyphon longiusculus kommt auch auf Rhodos, in der westlichen Türkei, in Israel und Georgien vor.

Neues Material: $20^{\star} o^{\star}$, IRAN, Prov. Mazandaran [IR08-23], Ramsar County, Elburz Mts., N-Slope, Eshkatechal, small stream, 1458 m, 06.VI.2008, 3650'53,0" N; 50³4'64,4" E, leg. A. Pütz, det. B. Klausnitzer. 


\section{Hydrocyphon segrex NYHOLM, 1972}

Das Areal dieser Art umfasst auch die Inseln Chios, Samos und Gökçeada sowie die westliche Türkei.

KLAusnitzer (1981): „Choramabad am Kaspischen Meer“. - „Choramabad“ (= Khorramabad) könnte Khorramabad südlich Tonekabon in der Provinz Māzandarān sein (FrISCH in litt.). Allerdings ist dieser Ortsname im Iran häufig und auch am Kaspischen Meer mehrfach vorhanden.

\subsection{Paläarktische Arten}

Das große Areal der folgenden Arten schließt auch den Iran ein. Meist liegen nur wenige Nachweise vor. Im Folgenden werden jeweils nur die einzelnen Provinzen angegeben.

\section{Cyphon laevipennis TOURNIER, 1868}

Bander Palevi am Kaspischen Meer (Klausnitzer 1981). „Bandar Pahlevi“ heißt heute „Bandar-e-Anzali“. Māzandarān, Āzarbāyjān-e Sharqi (KLAUSNITZER 1990).

Cyphon palustris C. G. Thomson, 1855

Māzandarān, Āzarbāyjān-e Sharqi, Kordestān (KLausnitzer 1990, 2012).

\section{Scirtes orbicularis (PANZER, 1793)}

Diese Art ist nicht über die gesamte Paläarktis verbreitet. Sie kommt in Europa mit Ausnahme der Iberischen Halbinsel und der Balkanhalbinsel bis zum Gebiet des Kaspischen Meeres und des Kaukasus vor (Klausnitzer 2009a). Der Fund im Iran (Bander Palevi am Kaspischen Meer, heute Bandar-e-Anzali) liegt an der Ostgrenze des Areals.

\section{Eine neue Art der Gattung Cyphon Paykull, 1799}

\section{Cyphon pareuoplus n. sp.}

Holotypus: ơ, IRAN, Prov. Mazandaran [IR08-17], Chalus County, Elburz Mts., N-Slope, 7 km N Makarud, small stream, Fagus forest, 1245 m, 03.VI.2008, 36³5'64,9" N; 51 09'55,4" E, leg. A. Pütz, in coll. Pütz.

Paratypen: $4 \sigma^{\star} \sigma^{\star}$, Fundortangaben wie Holotypus, $2 \sigma^{\star} o^{\star}$ in coll. PÜTZ, $2 \sigma^{\star} o^{\star}$ in coll. KLAUSNitzer. $10^{\star}$, IRAN, Prov. Mazandaran [IR08-22], Tonekabon County, Elburz Mts., N-Slope, 4,5 km SW Khanian, Sehezar forest, leaves debris, sifted, small stream, $942 \mathrm{~m}, 05 . \mathrm{VI} .2008,36^{\circ} 32^{\prime} 61,7^{\prime \prime} \mathrm{N}$; 5049'89,2" E, leg. A. Pütz, det. B. Klausnitzer, in coll. Klausnitzer. $10^{\star}$, IRAN, Prov. Mazandaran [IR08-04A], Sari County, Mohammadabad, Elburz Mts., N-Slope, $1 \mathrm{~km} \mathrm{~W}$ Afra Chal, small stream, leaves, sifted, 520 m, 30.V.2008, 36 $14^{\prime} 11,9^{\prime \prime} \mathrm{N} ; 53^{\circ} 13^{\prime} 61,0^{\prime \prime} \mathrm{E}$, leg. A. Pütz, det. B. Klausnitzer, in coll. Pütz. $10^{\star}$, IRAN, Prov. Mazandaran [IR08-23], Ramsar County, Elburz Mts., N-Slope, Eshkatechal, small stream, 1458 m, 06.VI.2008, 36 50'53,0" N; 50³4'64,4" E, leg. A. Pütz, det. B. Klausnitzer, in coll. Pütz.

Körperlänge (Länge Pronotum + Elytre) 2,85-3,04 mm. Körper länglich oval (Körperlänge/maximale Körperbreite $=1,67-1,78)$.

Kopf schwarzbraun; dicht und kräftig gekörnelt punktiert; hell nach vorn gerichtet behaart. Labrum, Mandibeln, Maxillarpalpen und Labialpalpen hellbraun. 1. bis 3. Antennenglied 
und Basis des 4. Gliedes hellbraun, übrige Antennenglieder braun bis dunkelbraun. Maße siehe Tabelle 2.

Pronotum in der Mitte braun, Seiten und Hinterrand hellbraun oder schwarzbraun, oder nur Seiten- und Hinterrand etwas aufgehellt; innerhalb der Hinterecken gerade; fein und dicht punktiert, Punktzwischenräume glatt (100fache Vergrößerung); hell nach außen gerichtet behaart. Maximale Breite 1,01-1,12 mm; Länge entlang der Mittellinie $0,50-0,53 \mathrm{~mm}$. Scutellum hellbraun, deutlich punktiert.

Elytren variabel: hellbraun, an der Basis, neben der Naht sowie am Apex nach außen gerichtet und am Seitenrand dunkelbraun längsgebändert (Fig. 1) oder nur um das Scutellum und am Seitenrand dunkelbraun oder Körper einfarbig schwarzbraun. Elytren mit deutlichen Rippen; dichter und gröber als das Pronotum punktiert, Punktzwischenräume glatt (100fache Vergrößerung); mit heller, nach hinten gerichteter Behaarung. Länge zwischen Schulter und Apex entlang der Körperlängsachse 2,35-2,51 mm; Breite einer Elytre in der Mitte 0,84-0,91 mm.

Beine hellbraun. Sternite braun, nach hinten gerichtet hell behaart. 7. Sternit flach dreieckig, hinten gerundet. Maximale Breite 0,85-0,91 mm; maximale Länge in der Mitte 0,28-0,30 mm.

Das 9. Sternit (Fig. 2) ist eine nach hinten gerundete Platte, deren Rand in einem schmalen Streifen verstärkt ist. Hinten befinden sich zahlreiche feine Borsten. Gesamtlänge des 9. Sternit 0,38-0,40 mm; maximale Breite der Platte 0,27-0,31 mm.

Das 8. Tergit (Fig. 3) besteht aus einer breiten, geschlossenen, hinten gerundeten Platte und annähernd geraden Bacilla lateralia. Die Platte ist am Hinterrand mit einem Saum spitzer Borsten bedeckt, davor befindet sich ein Feld mit kurzen Borsten. Gesamtlänge des 8. Tergit 0,400,45 mm; maximale Breite der Platte 0,34-0,38 mm; Länge der Bacilla lateralia 0,26-0,30 mm.

Die Platte des 9. Tergit ist hinten nur schwach sklerotisiert (Fig. 4), der Hinterrand ist mit einem Saum spitzer Mikrotrichen bedeckt. Die Bacilla lateralia sind schmal und vorn zugespitzt. Länge des 9. Tergit ca. 0,40-0,45 mm; maximale Breite 0,33-0,37 mm; Länge der Bacilla lateralia 0,20$0,25 \mathrm{~mm}$.

Tegmen (Fig. 5) mit einem kurz gestielten, verbreiterten Kapulus (0,07-0,08 mm), der in der Mitte einen schmalen sklerotisierten Stab enthält. Dieser gabelt sich und verstärkt die Parameren am Innenrand. Die Parameren sind mit kräftigen Borsten dicht bedeckt. Gesamtlänge 0,41$0,43 \mathrm{~mm}$; maximale Breite 0,27-0,28 $\mathrm{mm}$.

Penis (Fig. 6) mit einer rechteckigen, an den Seiten fast parallelen, vorn und am Rand verstärkten Pala. Aus dem Boden der Pala entspringt seitlich in der Mitte jeweils ein schmaler Stab. Dieser ist nach außen gebogen, vor dem Ende etwas erweitert (Fig. 6 Pfeil) und endet spitz. Er überragt die Prostheme. Diese sind glatt, verschmälern sich ausgehend von einer breiten Basis und sind vor allem hinten etwas nach innen gebogen (Fig. 7). Gesamtlänge des Penis 0,53-0,58 mm; maximale Breite der Pala 0,15-0,16 mm; Länge der Pala 0,32-0,36 mm; Länge der Prostheme 0,20-0,23 mm; Länge der Palastäbe 0,32-0,38 mm.

Weibchen unbekannt.

Areal: Cyphon pareuoplus n. sp. ist bisher nur von den Fundorten der Typen bekannt. 
Derivatio nominis: Der Artname „pareuoplus“, abgeleitet von $\pi \alpha \varsigma \dot{\alpha}=$ nahe bei (gr.) soll auf die Nähe zu Cyphon euoplus hinweisen.

\section{Differentialdiagnose:}

Auch bei Cyphon euoplus Nyнolm, 1970 finden sich in der Pala entspringende, nach hinten gerichtete Stäbchen, weshalb Cyphon pareuoplus n. sp. mit dieser Art verglichen wird (Tab. 3).

Tab. 3: Vergleich zwischen Cyphon euoplus (Fig. 7) und Cyphon pareuoplus n. sp. (Fig. 5, 6).

\begin{tabular}{|l|l|l|}
\hline \multicolumn{1}{|c|}{ Merkmal } & \multicolumn{1}{|c|}{ euoplus } & \multicolumn{1}{c|}{ pareuoplus n. sp. } \\
\hline $\begin{array}{l}\text { Basis der Fortsätze des Pala- } \\
\text { bodens }\end{array}$ & $\begin{array}{l}\text { in der Mitte der Pala entspringend } \\
\text { (Fig. 8) }\end{array}$ & $\begin{array}{l}\text { am Seitenrand der Pala entsprin- } \\
\text { gend (Fig. 6) }\end{array}$ \\
\hline Fortsätze des Palabodens & gerade, kürzer als Prostheme (Fig. 8) & $\begin{array}{l}\text { nach außen gebogen, länger als } \\
\text { Prostheme (Fig. 6) }\end{array}$ \\
\hline Querbrücke der Pala & breit und ungeteilt (Fig. 8) & in der Mitte unterbrochen (Fig. 7) \\
\hline Ansatz der Prostheme & etwas vom Hinterrand der Pala entfernt & direkt am Hinterrand der Pala \\
\hline Prostheme & stark nach innen gebogen (Fig. 8) & $\begin{array}{l}\text { mehr gerade, nur hinten nach } \\
\text { innen gebogen (Fig. 7) }\end{array}$ \\
\hline
\end{tabular}

\section{Eine neue Art der Gattung Elodes Latreille, 1796}

\section{Elodes unicolor n. sp.}

Holotypus: ơ, IRAN, Yasd Province, SW Taft: Dehbala, 2760 m, N 31³4'16", E $054^{\circ} 07^{\prime} 17^{\prime \prime}$, 17.05.2010, leg. Frisch \& Serri, in coll MFN.

Paratypen: 2 q + , IRAN, Kerman Province, pass Mahan - Sirch (Banan Mts.), 2800 m, N $30^{\circ} 12^{\prime} 07^{\prime \prime}$, E $057^{\circ} 24^{\prime} 22^{\prime \prime}, 20.05 .2010$, lg. Frisch \& Serri, 1 i in coll MFN. 1 i in coll. KLAUsNITZER.

Körperlänge (Länge Pronotum in der Mitte + Länge der Elytren zwischen Schulter und Apex entlang der Körperlängsachse): 5,41 mm ( $\overbrace{}^{*}) ; 5,77$ mm ( + ). Körper langgestreckt (Fig. 9). Index Körperlänge/Breite der Elytren = 2,37 ( $\left.\sigma^{*}\right) ; 2,38$ ( ( ). Körper einfarbig braun, Pronotum und Scutellum etwas heller.

Kopf rotbraun, zwischen den Augen hinten rechteckig, schwarzbraun ( $\overbrace{}^{\star})$ oder einfarbig braun ( ); gekörnelt punktiert; hell behaart, Haare nach vorn gerichtet. Mundwerkzeuge braun. 1. bis 3. Antennenglied braun, übrige Glieder schwarzbraun.

Pronotum mit runden Punkten dicht bedeckt; hell behaart. Länge entlang der Mittellinie 1,10 m (o); 1,09 mm ()); maximale Breite 1,63 mm (o); 1,62 mm (ㅇ). Scutellum dicht und rau wie die Elytren punktiert.

Elytren dicht und rau punktiert; Längsrippen deutlich ausgebildet; Elytren hell nach hinten gerichtet behaart. Breite einer Elytre in der Mitte 1,19 $\mathrm{mm}\left(0^{*}\right) ; 1,21 \mathrm{~mm}($ ( $)$; Länge einer Elytre zwischen Schulter und Apex entlang der Körperlängsachse 4,31 mm ( $\left.0^{\star}\right) ; 4,68 \mathrm{~mm}$ ( ( +).

Beine braun. Sternite schwarzbraun ( $\left.o^{\star}\right)$ bis braun ( $(9)$.

Männchen:

7. Sternit hinten halbkreisförmig eingebuchtet (Fig. 10). Buchttiefe 0,06 mm; Länge neben der Bucht 0,61 mm; Breite 1,34 mm. 
7. Tergit eine breite Platte bildend, mit geschwungenen Bacilla lateralia (Fig. 11). An der Eintrittstelle der Bacilla lateralia in die Platte befindet sich eine längliche ösenförmige, wenig sklerotisierte Struktur. Länge in der Mitte 0,52 mm; Breite der Platte ca. 1,10 mm; Bacilla lateralia (ohne Berücksichtigung der Krümmung) 0,48 mm lang.

8. Sternit (Fig. 12) mit großen, hinten gerundeten Pterygien. Stiel schmal, stabförmig sklerotisiert und in die Basis der Pterygien hineinragend und dort verbreitert dreispitzig endend (Fig. 12 Pfeil). Vorher gabelt sich der Stiel seitlich und ragt schräg in die Pterygien hinein. Gesamtlänge des 8. Sternit 0,88 mm; Länge des Stiels 0,62 mm; maximale Breite 0,68 mm.

9. Sternit (Fig. 13) hinten mit einem Feld langer Borsten. Der beborstete Teil ist abgesetzt und $0,25 \mathrm{~mm}$ lang. Seitenrand verstärkt, vorn sind die sklerotisierten Ränder durch eine feine Membran miteinander verbunden. Gesamtlänge des 9 . Sternit $1,16 \mathrm{~mm}$; maximale Breite 0,41 mm.

8. Tergit (Fig. 14) mit einer breiten Platte, deren hinterer Teil abgesetzt erscheint und mit Mikrotrichen besetzt ist. Der Hauptteil der Platte ist mit kurzen Borsten besetzt. Bacilla lateralia fast gerade. Gesamtlänge des 8 . Tergit $0,94 \mathrm{~mm}$; Breite der Platte $0,59 \mathrm{~mm}$; Länge der Bacilla lateralia $0,75 \mathrm{~mm}$.

9. Tergit mit sklerotisierten, zunächst geraden, hinten nach innen gerichteten Bacilla lateralia, die sich beim Eintritt in die Platte schwach gabeln (Fig. 15), Platte hinten wenig sklerotisiert. Gesamtlänge des 9. Tergit ca. 0,92 mm; Breite der Platte 0,52 mm; Länge der Bacilla lateralia (ohne Berücksichtigung der Krümmung) 0,74 mm.

Tegmen (Fig. 16) mit geraden, an der Basis etwas breiteren, schlanken, hinten zugespitzten Parameren. Gesamtlänge des Tegmen 1,92 mm; maximale Breite 0,28 mm. Lateralgriffel schmal $(0,02 \mathrm{~mm})$ und $0,43 \mathrm{~mm}$ lang.

Penis schlank (Fig. 17), Parameroide hinten mit einem nach vorn gerichteten spitzen Seitenzahn (bis zur Spitze 0,14 mm lang), hinten mit Sinnesporen dicht bedeckt, davor befinden sich kurze Borsten. Gesamtlänge des Penis 1,89 mm; maximale Breite in der Mitte 0,19 mm.

\section{Weibchen:}

7. Sternit hinten gerundet. In der Mitte des Hinterrandes befindet sich eine kleine Spitze, die sich nach vorn in einen $0,18 \mathrm{~mm}$ langen Kiel fortsetzt. Länge maximal 0,62 mm; Breite 1,38 mm.

Das 8. Sternit bildet eine geschlossene Platte, die hinten gespalten ist (Fig. 18) und dort einen Saum von Mikrotrichen trägt. Im Inneren befindet sich jederseits ein sklerotisiertes Stäbchen, auf dem einige kurze Borsten entspringen. Gesamtlänge des 8 . Sternit 1,00 mm; maximale Breite 0,52 mm.

8. Tergit (Fig. 19) mit einer wenig sklerotisierten Platte und kräftigen, etwas gebogenen Bacilla lateralia. Der Hinterrand der Platte trägt einen Saum von Mikrotrichen, davor befinden sich einzelne kurze Borsten. Gesamtlänge des 8 . Tergit $1,38 \mathrm{~mm}$; Breite der Platte 0,61 mm; Länge der Bacilla lateralia (ohne Berücksichtigung der Krümmung) 1,02 mm.

Das Bursalsklerit besteht aus mehreren sklerotisierten Längsfalten, von denen die drei mittleren am kräftigsten sind (Fig. 20). Länge ca. 1,00 mm; Breite 0,26 mm.

Areal: Bisher nur von den Fundorten der Typen bekannt.

Derivatio nominis: Der Artname „unicolor“ bezieht sich auf die Einfarbigkeit des Körpers und ist abgeleitet von unicolor $=$ einfarbig (lat.).

\section{Differentialdiagnose:}

Nach dem Bau der Tegmen (Parameren einfach zugespitzt), des 8. Sternit (Stiel gegabelt, dazwischen, an der Basis der Bucht \pm sklerotisiert) und des 8 . Tergit (hinten ganzrandig) kann Elodes unicolor n. sp. der Elodes sericea-Gruppe zugeordnet werden (zur Definition siehe KLAusNitzer 
2009b). Bisher sind sechs Arten aus dieser Gruppe bekannt, deren Gesamtareal von der östlichen Mediterraneis bis Mittelasien reicht.

Wenn man die Form der Parameroide zur Differenzierung der Arten an die erste Stelle setzt, so ist die neue Art mit Elodes eberti Klausnitzer, 1970, E. orientalis Iablokoff-Khnzorian, 1973 und E. persica Klausnitzer, 1975 zu vergleichen (Tabelle 4). Alle diese Arten sind auch oder ausschließlich aus dem Iran bekannt. Unterschiede ergeben sich vor allem in den folgenden drei Merkmalen:

- die Parameroide sind hakenförmig (Fig. 17).

- das Fehlen einer Erweiterung auf der Innenseite der Protibien der Männchen (Fig. 9).

- Körper einfarbig (Fig. 9).

Bisher ist nur von E. eberti das Bursalsklerit bekannt. Es ist \pm kompakt und besteht aus zwei Teilen. Dasjenige von E. unicolor n. sp. zeigt mehrere sklerotisierte Längsfalten. Sein Bau lässt es als fraglich erscheinen, ob die kompakte Form und die Zweiteiligkeit als ein gemeinsames Merkmal der Elodes sericea-Gruppe angesehen werden kann. Allerdings sind neben den Genannten nur noch von E. jelineki Klausnitzer, 2009 und E. sericea Kiesenwetter, 1859 die Weibchen bekannt, die das bisher als artengruppenspezifisch angesehene Bild des Bursalsklerit zeigen.

Tab. 4: Vergleich ( $\left.\sigma^{\top} \sigma^{\star}\right)$ von Elodes unicolor n. sp. mit E. eberti KLausnitzer, 1970, E. orientalis IablokoffKhnzorian, 1973 und E. persica Klausnitzer, 1975. Durchschnittswerte (Ø): unicolor $\mathrm{n} . \mathrm{sp} . \mathrm{n}=1$, eberti $\mathrm{n}=6$, orientalis $\mathrm{n}=3$, persica $\mathrm{n}=3$. Messwerte nach KLAusnitzer (2009b), ergänzt. Alle Maße in mm.

\begin{tabular}{|c|c|c|c|c|}
\hline Merkmal & unicolor $\mathrm{n} . \mathrm{sp}$. & eberti & orientalis & persica \\
\hline Körperlänge & 5,41 & $4,30-5,00$ & $\begin{array}{l}4,40-5,22 ; \\
\varnothing 4,74\end{array}$ & $4,75-5,15$ \\
\hline Protibia nach innen erweitert & nein & ja & ja & ja \\
\hline Elytren, Färbung & einfarbig & mehrfarbig & mehrfarbig & mehrfarbig \\
\hline Parameroide, Form & hakenförmig & breit blattförmig & lang, schmal & messerförmig \\
\hline \begin{tabular}{|l}
$\begin{array}{l}\text { Parameroide, Länge hinter dem } \\
\text { Lateralzahn }\end{array}$ \\
\end{tabular} & 0,14 & $0,13-0,15$ & $0,08-0,13$ & $\begin{array}{l}0,10-0,12 ; \\
\varnothing 0,11\end{array}$ \\
\hline Länge Penis & 1,89 & $1,74-1,90 ; \varnothing 1,82$ & $\begin{array}{l}1,80-1,90 ; \\
\varnothing 1,86\end{array}$ & $\begin{array}{l}1,91-2,03 ; \\
\varnothing 1,96\end{array}$ \\
\hline Parameren & gerade & kurz, gebogen & gerade & gerade \\
\hline Länge Tegmen & 1,92 & $1,39-1,41 ; \varnothing 1,40$ & $\begin{array}{l}1,47-1,60 ; \\
\varnothing 1,53 \\
\end{array}$ & $\begin{array}{l}1,55-1,80 ; \\
\varnothing 1,64\end{array}$ \\
\hline 7. Sternit, Tiefe Bucht & 0,06 & 0,08-0,09; Ø 0,09 & $\begin{array}{l}0,11-0,14 ; \\
\varnothing 0,12\end{array}$ & $\begin{array}{l}0,07-0,11 ; \\
\varnothing 0,09\end{array}$ \\
\hline 8. Sternit, maximale Länge & 0,88 & $0,95-1,03 ; \varnothing 1,00$ & $\begin{array}{l}0,72-0,82 ; \\
\varnothing 0,77\end{array}$ & $\begin{array}{l}0,86-0,93 ; \\
\varnothing 0,90\end{array}$ \\
\hline 8. Sternit, Pterygien mit Zähnchen & nein & ja & nein & nein \\
\hline $\begin{array}{l}\text { 8. Sternit, Stiel an der Basis der } \\
\text { Bucht } \pm \text { sklerotisiert und verbreitert }\end{array}$ & ja & nein & nein & ja \\
\hline Areal & Iran & \begin{tabular}{|l|} 
Ukraine, östliche Türkei, \\
Aserbaidschan, Iran
\end{tabular} & $\begin{array}{l}\text { Tadschiki- } \\
\text { stan, Iran }\end{array}$ & Iran \\
\hline
\end{tabular}

\section{Dank}

Die Möglichkeit zur Untersuchung der interessanten Tiere aus dem Iran verdanke ich vor allem den Herren Dr. Claude Besuchet, Muséum d'Histoire naturelle Genève, Dr. Johannes Frisch und Bernd Jäger, Museum für Naturkunde Berlin, Dipl.-Biol. Matthias Hartmann, Naturkundemuseum Erfurt, Dr. Josef 
JelíneK, Entomologické oddelení, Nationalmuseum Praha, und Andreas Pütz, Eisenhüttenstadt, wofür ich sehr herzlich danke. Herr Peter Schüle, Herrenberg, zeichnete die Figuren 2 bis 8 und 10 bis 20 nach den Vorlagen des Verfassers, die Figuren 1 und 9 fertigte Herr Lutz Behne, Senckenberg Deutsches Entomologisches Institut Müncheberg an, wofür ich ebenfalls sehr herzlich danke. Herrn Dr. LothaR Zerche vom gleichen Institut sowie Herrn Dr. Johannes Frisch, Museum für Naturkunde Berlin, danke ich für wichtige Hinweise zum Manuskript.

\section{Literatur}

Klausnitzer, B. 1970: Zur Kenntnis der Gattung Helodes Latreille (Coleoptera, Helodidae). Entomologische Nachrichten 14 (12): 177-184.

Klausnitzer, B. 1972: Zur Kenntnis der Gattung Helodes Latreille (Coleoptera, Helodidae). 2. Fortsetzung. - Entomologische Nachrichten 16 (4): 29-33.

Klausnitzer, B. 1975: Zwei neue Arten der Gattung Helodes Latreille aus der Palaearktis (Coleoptera: Helodidae). - Beiträge zur Entomologie 25 (2): 329-333.

KLAusnitzer, B. 1981: Zur Kenntnis der Cyphon- und Hydrocyphon-Fauna des Iran (Insecta, Coleoptera, Helodidae). - Faunistische Abhandlungen Staatliches Museum für Tierkunde in Dresden 8 (4): 63-65.

Klausnitzer, B. 1990: Anmerkungen zur Helodidenfauna des Iran (Coleoptera). - Entomologische Nachrichten und Berichte 34 (4): 159-165.

Klausnitzer, B. 2006: Family Scirtidae Fleming, 1821. - In: Löbl, I. \& Smetana, A. (Eds.): Catalogue of Palaearctic Coleoptera. Volume 3. - Apollo Books, Stenstrup: 316-323.

KLAusnitzer, B. 2008: Neufunde von Scirtidae (Coleoptera) aus der Westpaläarktis und Ergänzungen zum „Catalogue of Palaearctic Coleoptera. Volume 3 (Scirtidae)“ I. - Entomologische Nachrichten und Berichte 52 (3-4): 203-206.

KLAusnitzer, B. 2009a: Insecta: Coleoptera: Scirtidae. (Die Scirtidae der Westpaläarktis). - In: Süßwasserfauna von Mitteleuropa. Begründet von A. BraUer, herausgegeben von P. Zwick. Band 20/17. Spektrum Akademischer Verlag, Heidelberg: 326 S., 1041 Abb., 8 Farbtaf.

Klausnitzer, B. 2009b: Contribution to the knowledge of Elodes sericea species-group with description of two new species of the genus Elodes Latreille, 1796 from Middle Asia (Coleoptera, Scirtidae). - Acta Entomologica Musei nationalis Pragae 49 (2): 711-728.

KLAusnitzer, B. 2010: Neufunde von Scirtidae (Coleoptera) aus der Westpaläarktis und Ergänzungen zum „Catalogue of Palaearctic Coleoptera. Volume 3 (Scirtidae)“ II. - Entomologische Nachrichten und Berichte 54 (1): 25-30.

KLausnitzer, B. 2012: Neufunde von Scirtidae (Coleoptera) aus der Westpaläarktis und Ergänzungen zum „Catalogue of Palaearctic Coleoptera. Volume 3 (Scirtidae)“ III. - Entomologische Nachrichten und Berichte 56 (2): 147-154.

Klausnitzer, B., im Druck: Anmerkungen zu den Scirtidae (Coleoptera) Armeniens. - Entomologische Nachrichten und Berichte 56 (3-4).

Lattin, G. De 1967: Grundriß der Zoogeographie - Gustav Fischer Verlag Jena: 602 S.

Nyнolm, T. 1970: Beiträge zur Kenntnis der paläarktischen Helodiden. 1. Cyphon-Arten aus der Türkei. Studien über die Familie Helodidae. XI. - Entomologisk Tidskrift 91: 24-41.

Nyнolm, T. 1976: Neue paläarktische Arten der Gattung Cyphon Paykull. V. (Coleoptera, Helodidae). Studien über die Familie Helodidae. XVII. - Entomologica scandinavica 7: 283-292.

\section{Anschrift des Verfassers:}

Prof. Dr. sc. nat. Dr. rer. nat. h. c. Bernhard Klausnitzer

Mitglied des Senckenberg Deutschen Entomologischen Instituts

Lannerstraße 5, 01219 Dresden

E-Mail: klausnitzer.col@t-online.de

\section{Subject editor:}

Dr. L. Zerche 


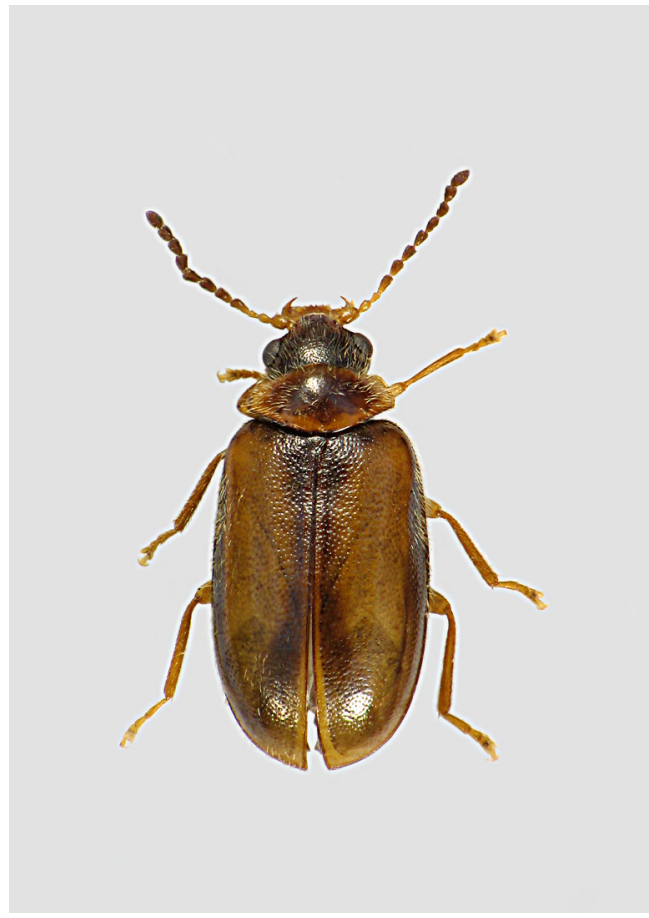

Fig. 1: Cyphon pareuoplus n. sp., Habitus, dorsal.

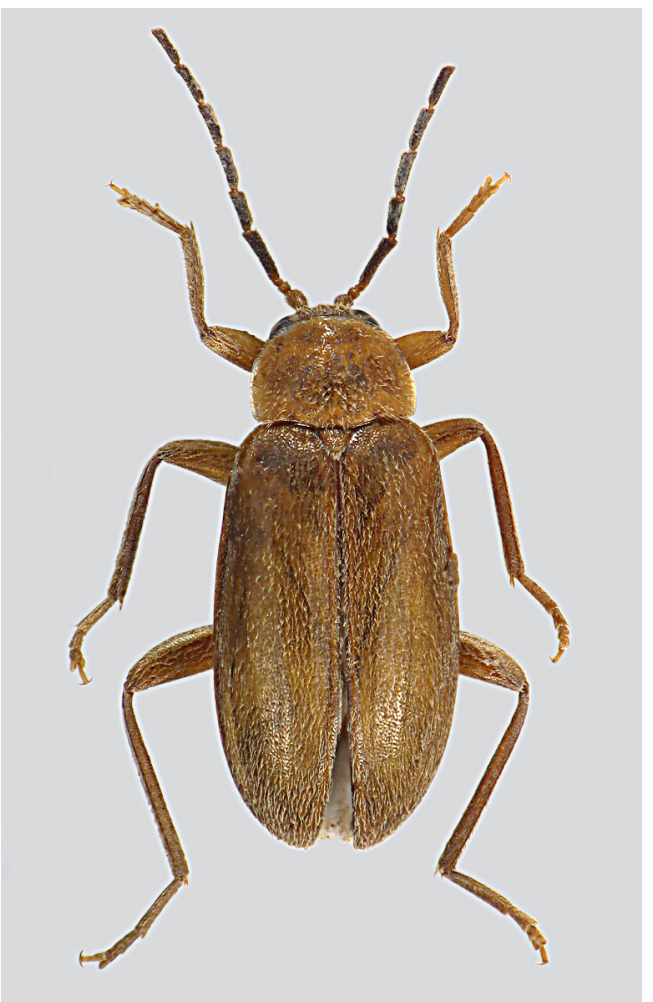

Fig. 9: Elodes unicolor n. sp., ơ, Habitus, dorsal. 

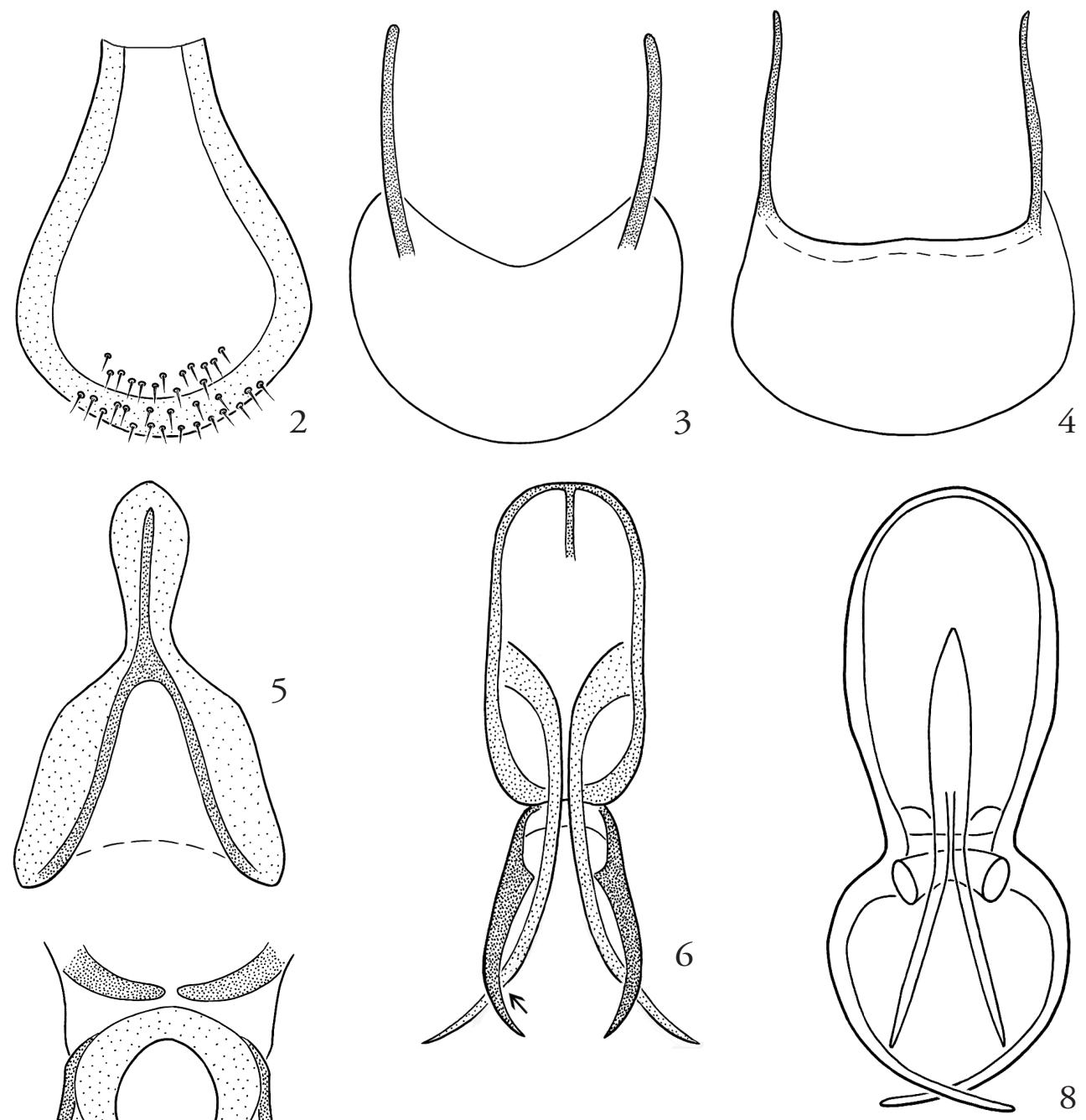

Fig. 2-7: Cyphon pareuoplus n. sp., 2: 9. Sternit, 3: 8. Tergit, 4: 9. Tergit, 5: Tegmen, 6: Penis, 7: Penis, Prostheme.

7 Fig. 8: Cyphon euoplus, Penis (umgezeichnet nach Nyноцм 1970). 

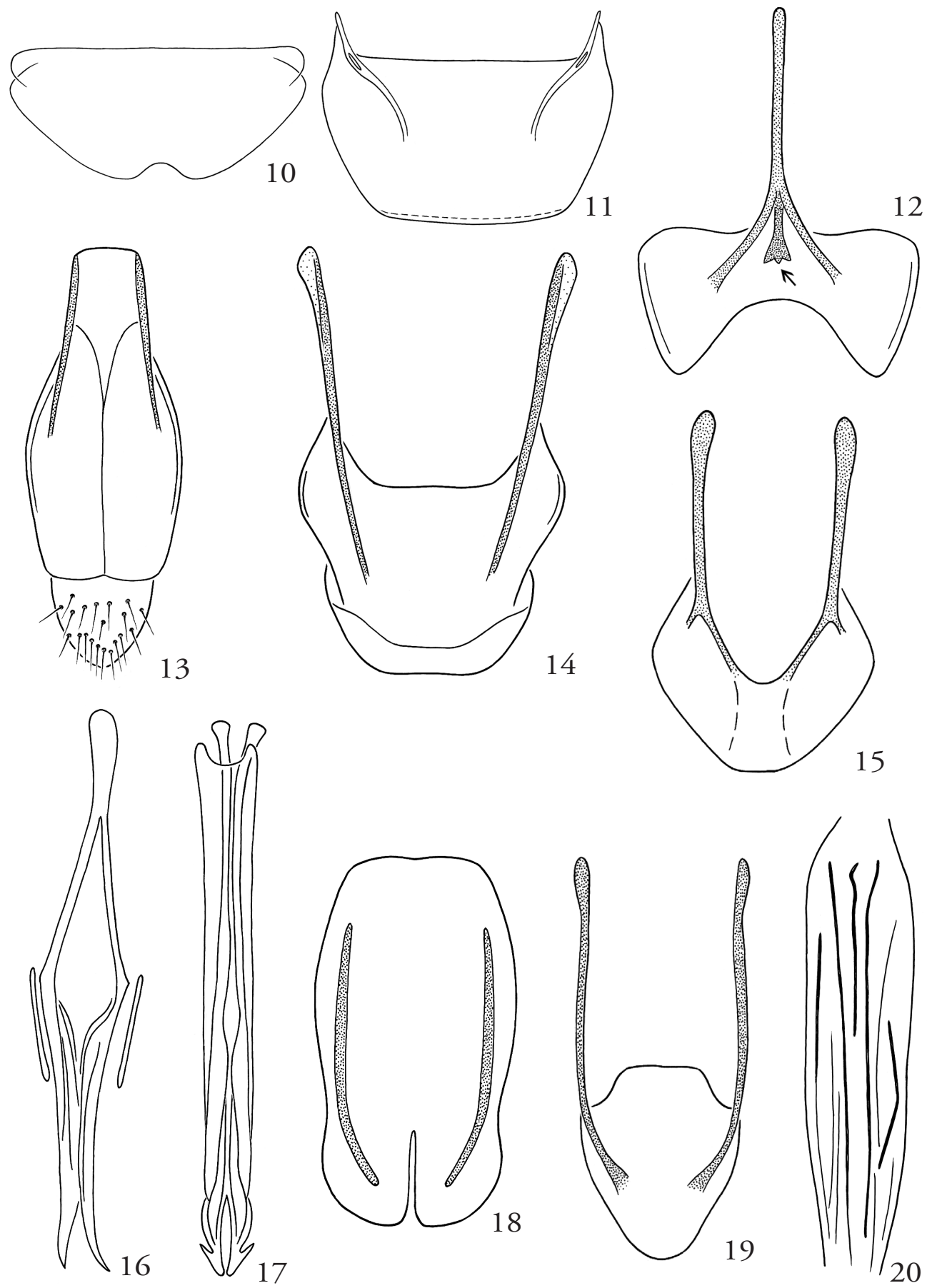

Fig. 10-17: Elodes unicolor n. sp. ơ, 10: 7. Sternit, 11: 7. Tergit, 12: 8. Sternit, 13: 9. Sternit, 14: 8. Tergit, 15: 9. Tergit (Hinterrand im Präparat artefiziell etwas nach vorn umgeklappt), 16: Tegmen, 17: Penis. Fig. 18-20: Elodes unicolor n. sp. ㅇ, 18: 8. Sternit, 19: 8. Tergit. Fig. 20: Bursalsklerit. 